\title{
A study of the near field atmospheric dispersion of emissions at height: Comparison of Gaussian plume models (Doury, Pasquill-Briggs, Caire) with krypton 85 measurements taken around La Hague nuclear reprocessing plant
}

\author{
D. Maro, B. Crabol ${ }^{1}$, P. Germain, Y. Baron ${ }^{2}$, D. Hebert and P. Bouisset ${ }^{3}$
}

Institut de Protection et de Sûreté Nucléaire, Département de PRotection de l'Environnement, Service d'Études et Recherches Radioécologiques dans les Milieux NATurels, LERFA, 50130 Cherbourg-Octeville, France

${ }^{1}$ Institut de Protection et de Sûreté Nucléaire, Département de Protection de l'Environnement, CE-Saclay, bâtiment 130, 91191 Gif-sur-Yvette, France

${ }^{2}$ Marine Nationale, Groupe d'Études Atomiques (GEA), BP. 34, 50115 Cherbourg Naval, France

${ }^{3}$ Institut de Protection et de Sûreté Nucléaire, Département de Protection de l'Environnement, LMRE, Bois des Rames, bâtiment 501, 91400 Orsay, France

\begin{abstract}
Gaussian atmospheric dispersion models are principally validated for release at ground level, or, if the release is very high up, at distances sufficiently removed from the source for the plume to have reached the ground. In order to improve the evaluation of the near field dispersion $(<4 \mathrm{~km})$ for above-ground releases, the Institute for Protection and Nuclear Safety (IPSN) began an in situ study in June 1997 around the La Hague nuclear reprocessing plant using krypton $85\left({ }^{85} \mathrm{Kr}\right.$, released in the gaseous effluent by a stack of $\left.100 \mathrm{~m} \mathrm{high}\right)$ as a plume tracer. The aim of this study is to compare the results for the atmospheric transfer coefficients (ATC) obtained in ${ }^{85} \mathrm{Kr}$ measurement surveys with the results of three Gaussian atmospheric dispersion models (Doury, Pasquill-Briggs and CAIRE) in order to define the distances at which corrections need to be made to the models and thus improve determination of the ATCs. For all the results, it is observed as expected that the models are not applicable for the source near field; however, the study clearly shows that the CAIRE model gives the best results at all distances.
\end{abstract}

\section{INTRODUCTION}

The Gaussian atmospheric dispersion models traditionally used for operational purposes (mainly Doury and Pasquill-Briggs) are principally validated for release at ground level (or close enough to approximate to ground level), or, if the release is very high up, at distances sufficiently removed from the source for the plume to have reached the ground [1] [2] [3].

In order to improve the evaluation of the near field dispersion $(<4 \mathrm{~km})$ for above-ground releases, the Institute for Protection and Nuclear Safety (IPSN) in collaboration with the French Navy's Groupe d'Etudes Atomiques began an in situ study in June 1997 [4] around the La Hague nuclear reprocessing plant using as a plume tracer krypton $85\left({ }^{85} \mathrm{Kr}\right.$ ), a inert gas released in the gaseous effluent (from a release stack $100 \mathrm{~m}$ high).

${ }^{85} \mathrm{Kr}$, which is a $\beta^{-}$and $\gamma$ emitter radioactive isotope with a half-life of 10.71 years, is produced naturally and in nuclear reactors [5] [6]. It has also been released into the atmosphere following nuclear explosions, but currently its main source is release by nuclear reprocessing plants such as La Hague.

The continuous measurement of ${ }^{85} \mathrm{Kr}$ activity in the air for different distances from the point of release at the La Hague plant and for a variety of weather conditions provides a useful tool for validating atmospheric dispersion models for releases at altitude. It can be used to follow the passage of each release of ${ }^{85} \mathrm{Kr}$ and to calculate the atmospheric transfer coefficients (ATC).

The aim of this study is to compare the results for the ATCs obtained using ${ }^{85} \mathrm{Kr}$ measurement surveys around the La Hague COGEMA plant with the results of three Gaussian atmospheric dispersion models, Doury [1], Pasquill-Briggs [2] [3] and CAIRE [7], in order to define the distances for which corrections need to be made to the models and thus improve determination of the ATCs. The Doury model is mostly used in France and the Pasquill-Briggs model is used in Britain and the US. The CAIRE model, based on the Pasquill-Briggs model, is used by the French Navy. 


\section{MATERIALS AND METHODS}

\section{$2.1{ }^{85} \mathrm{Kr}$ field measurements around the La Hague nuclear reprocessing plant}

The IPSN carried out a series of fourteen ground measurement surveys for ${ }^{85} \mathrm{Kr}$ around the La Hague nuclear reprocessing plant (figure 1) from June 1997 to April 1998 for a range of different distances and weather conditions [4].

The field measurements were performed at distances between 575 and 4500 meters from the release points with wind speeds at 100 meters altitude above the La Hague plateau in the range 4.5 to 16.9 m.s. The diffusion weather conditions during the samples were normal diffusion in the Doury classification or neutral or slightly unstable in the Pasquill-Briggs classification (classes $\mathrm{C}$ and $\mathrm{D}$ ).

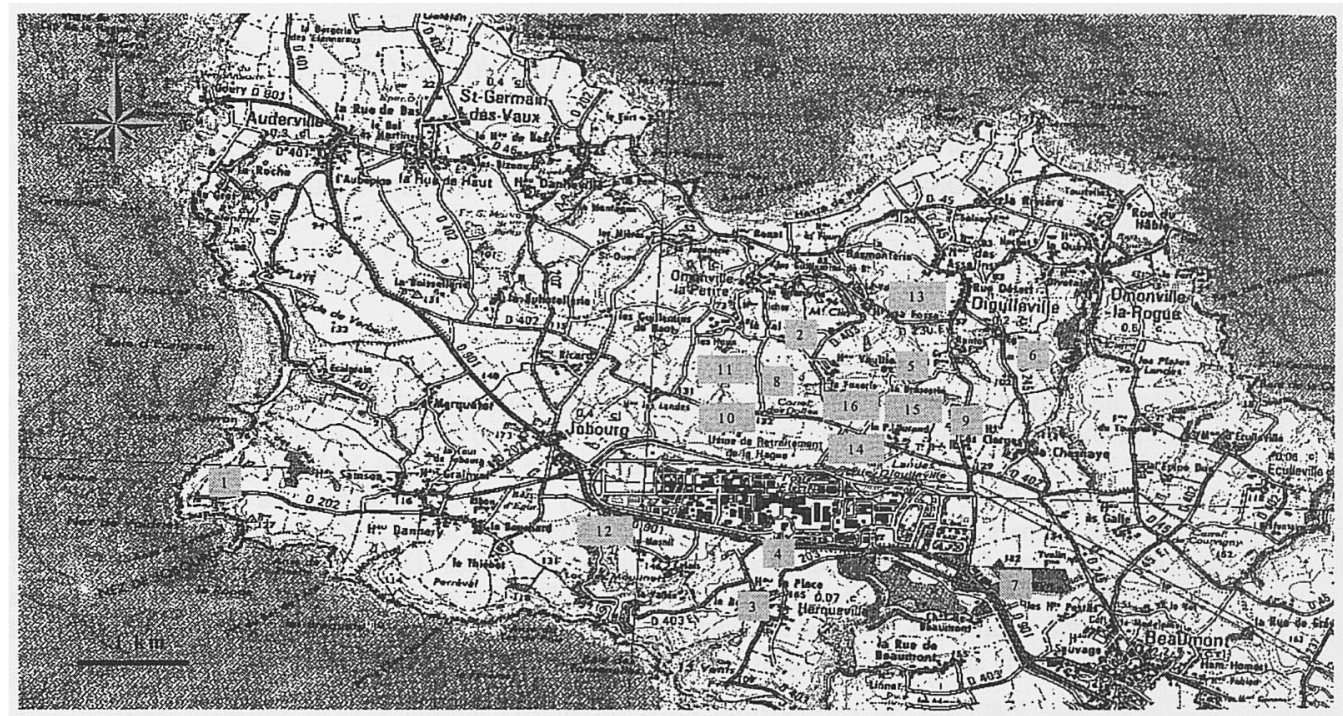

Figure 1: Survey areas for the different measurement campaigns (16 different measurement sites)

The measurement results enabled the ATCs on the ground to be calculated for the distances from the release point and for the weather conditions encountered during these measurement surveys. For the La Hague nuclear reprocessing plant, it is assumed that the ATC can be deduced from the ratio between the integral of the concentration measured in the environment during the shearing period $(\sim 30$ minutes $)$ and the quantity released during shearing. Figure 2 shows that this approximation is justified since most of the plume corresponding to shearing can be detected before the appearance of the plume caused by the next shearing. This leads to the following method of determining the ATC:

$$
A T C=\frac{\int_{t_{0}}^{t_{1}} X(M, t) \cdot d t}{\int_{t^{\prime}{ }_{0}}^{t_{1}^{\prime}} q(t) \cdot d t}
$$

where:

$-\mathrm{X}(\mathrm{M}, \mathrm{t})$ : Activity concentration at measurement point $(\mathrm{M})$ at time $\mathrm{t}\left(\mathrm{Bq} \cdot \mathrm{m}^{-3}\right)$,

$-q(t)$ : Source activity rate $\left(B q . s^{-1}\right)$,

$-t^{\prime} 0, t^{\prime} 1$ : Emission source(s) start and end times,

-t0, t1: Measurement(s) start and end times. 


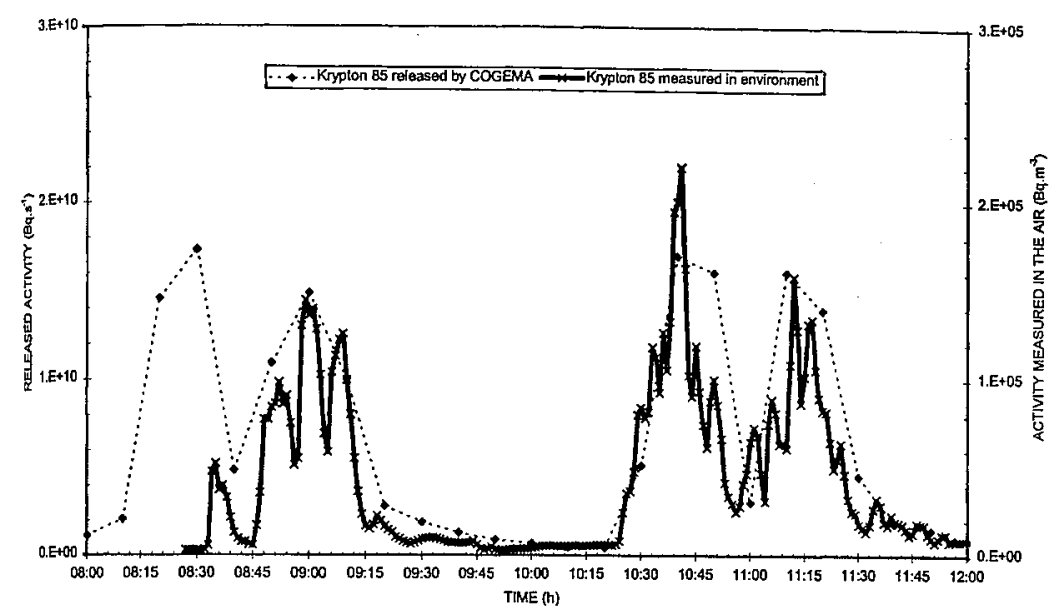

Figure 2: Example of ${ }^{85} \mathrm{Kr}$ environment measurements (measurements taken 20/01/1998 at Herqueville)

\subsection{Gaussian atmospheric dispersion models (Doury, Pasquill-Briggs and CAIRE)}

The radioactive elements released into the atmosphere are blown in the wind direction and dispersed in the mass of air under the action of natural atmospheric phenomena: transport and diffusion. Transport involves the displacement of the center of mass of the cloud between two given instants, while diffusion corresponds to the relative displacement of the component volumes within the cloud relatively to their center of gravity [8]. Several types of atmospheric dispersion models have been developed, including Gaussian models. Gaussian dispersion models are based on the solution of the general equation for diffusion transport of a passive gas, such as ${ }^{85} \mathrm{Kr}$, in a turbulent environment. If the concentration does not depend on time and if the flux of pollutant transported by the wind is high relative to the turbulent flux in the same direction, which in practice means a wind speed greater than $2 \mathrm{~m} \cdot \mathrm{s}^{-1}$, this gives the solution known as a Gaussian plume:

$A T C=\left\{\frac{1}{(2 \pi) \bar{U} \sigma_{h} \sigma_{z}} \exp \left(-\frac{\left(y-y_{0}\right)^{2}}{2 \sigma_{h}^{2}}\right)\right\} \times\left\{\exp \left(-\frac{\left(z-z_{0}\right)^{2}}{2 \sigma_{z}^{2}}\right)+\exp \left(-\frac{\left(z+z_{o}\right)^{2}}{2 \sigma_{z}^{2}}\right)\right\}$

where: - $y, z$ : coordinates of the observation point ( $\mathrm{x}$ taken to be in the average wind direction), $\mathrm{y}$ sideways and $z$ vertically,

$-y_{0}, z_{0}$ : coordinates of the release point,

- $\bar{U}$ : average wind speed,

$-\sigma_{h}, \sigma_{z}:$ standard deviations for the Gaussian distribution of the pollutant relative to its average location at time $\mathbf{t}$.

This is the equation used to make calculations for comparison with the measurements because the wind speed was high enough for the Gaussian plume hypothesis to hold true (wind speed $>2 \mathrm{~m} . \mathrm{s}^{-1}$ ).

The Doury model

Doury [1] distinguishes two categories of atmospheric stability: the normal diffusion class (average or strong turbulence) and the low diffusion class (weak turbulence). He considers that only the vertical distribution of the pollutant is affected by the stability of the atmosphere.

Normal atmospheric diffusion, or neutral to unstable atmosphere, corresponds to a vertical temperature gradient of less than or equal to $-0.5^{\circ} \mathrm{C} / 100 \mathrm{~m}$. Weak atmospheric diffusion, or stable atmosphere, corresponds to a vertical temperature gradient value greater than $-0.5^{\circ} \mathrm{C} / 100 \mathrm{~m}$. The standard deviations for the Doury model vary with the time elapsed since the instant of emission. 


\section{The Pasquill-Briggs model}

The standard deviations in the Pasquill-Briggs model [2] [3] are a function of the distance travelled by the pollutant from the source. Also, Pasquill-Briggs distinguishes six categories of atmospheric stability, which acts on both vertical and horizontal diffusion. The model uses six classes to describe the stability of the atmosphere: $\mathrm{A}=$ very unstable, $\mathrm{B}=$ moderately unstable, $\mathrm{C}=$ slightly unstable, $\mathrm{D}=$ neutral, $\mathrm{E}=$ stable and $\mathrm{F}=$ very stable.

\section{The CAIRE model}

The CAIRE model [7] is used by the French Navy for chronic releases or those occurring in accident situations. This model is designed for the near field, as it can only be used within a radius of $2000 \mathrm{~m}$ from the source point. This Gaussian model is based on the Pasquill-Briggs stability classes.

\section{RESULTS AND DISCUSSION}

\subsection{Atmospheric transfer coefficients measured at La Hague}

For the ${ }^{85} \mathrm{Kr}$ measurement surveys, the ATCs obtained vary from $8.110^{-7} \pm 2.110^{-7}$ to $7.810^{-6} \pm 2.010^{-6}$ s. $\mathrm{m}^{-3}$ for distances between 575 and $4500 \mathrm{~m}$ from the release points. The wind speed at $100 \mathrm{~m}$ varied between surveys within the range 4.5 to $16.9 \mathrm{~m} . \mathrm{s}^{-1}$ [4].

3.2 Comparison of ATCs calculated using the models with ATCs determined from ${ }^{85} \mathrm{Kr}$ field measurements

Figure 3 shows the comparison between the experimental ATC and the modeled ATC against distance from the release point.

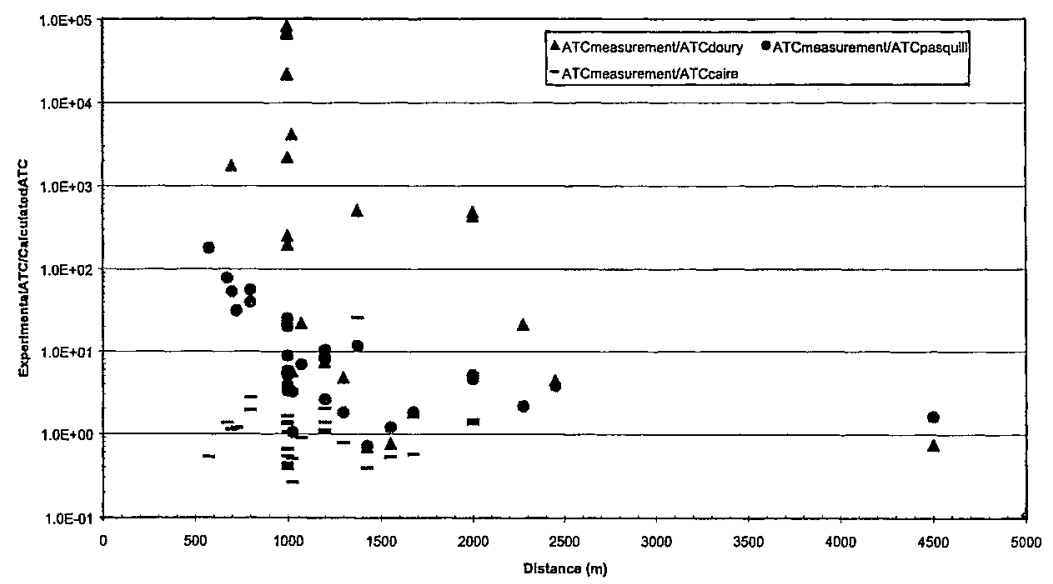

Figure 3: Comparison of ATCs calculated using the models with ATCs determined from ${ }^{85} \mathrm{Kr}$ field measurements

It can be observed that the experimental ATC determined from ${ }^{85} \mathrm{Kr}$ measurements is greatly undervalued by the models, both Doury and Pasquill-Briggs, at low distances from the source. The smaller the distance between the source point and the calculation point, the greater the deviation between the models and the measurements. This happens because the classical gaussian models, such as Doury and Pasquill-Briggs models, are not valid in the near field for releases from elevated sources, as is the case for the La Hague nuclear reprocessing plant. To correct this problem, some authors recommend applying alternative methods [9] [10] for the near field without any real physical basis, for example reducing the height of the release point arbitrarily. 
With the CAIRE model, the differences between the calculation results and the measurements are between $2.610^{1}$ and $310^{-1}$. Except for two cases, the differences between the model results and the measurements are less than a factor of 3 .

In this comparison between the results from the different models with ${ }^{85} \mathrm{Kr}$ field measurements for medium to strong turbulence (normal diffusion for Doury, classes C and D for Pasquill-Briggs), it is clear that the CAIRE model gives the best results. However, it should be mentioned that the CAIRE model is a near field model, not applicable beyond 2 kilometers from the source.

\section{CONCLUSION}

Fourteen ${ }^{85} \mathrm{Kr}$ measurement surveys were made around the La Hague nuclear reprocessing plant between June 1997 and April 1998 [4].

For these ${ }^{85} \mathrm{Kr}$ measurement surveys, the ATCs obtained vary:

- from $8.110^{-7} \pm 2.110^{-7}$ for a distance of $1000 \mathrm{~m}$ from the release point with a wind speed of $16.5 \mathrm{~m} . \mathrm{s}^{-1}$

- to $7.810^{-6} \pm 2.010^{-6} \mathrm{~s} . \mathrm{m}^{-3}$ for a distance of $1000 \mathrm{~m}$ from the release point with a wind speed of $11.1 \mathrm{~m} \cdot \mathrm{s}^{-1}$.

The comparison between the results from the different models (Doury, Pasquill-Briggs and CAIRE) with ${ }^{85} \mathrm{Kr}$ field measurements for medium to strong turbulence (normal diffusion for Doury, classes $\mathrm{C}$ and $D$ for Pasquill-Briggs) show clearly that the CAIRE model gives the best results. However, it is to be noted that this model is designed for the near field, as it can only be used within a radius of $2000 \mathrm{~m}$ from the source point.

Future work will be carried with ${ }^{85} \mathrm{Kr}$ measurements at ground level and in altitude for different atmospheric stability classes, including weak diffusion and over a range of distances from 200 to $4000 \mathrm{~m}$ from the release point [11]. This set of measurement results will be used to describe the actual vertical and horizontal plume distributions, then to extend the scope of application of Gaussian atmospheric dispersion models to situations such as the one of la Hague.

\section{Acknowledgements}

The authors would like to thank Ms Fitamant and Messrs. Le Bar, Cadioux and Arnaud from COGEMA La Hague for their help in carrying out the measurements and for providing us with weather data and ${ }^{85} \mathrm{Kr}$ release values.

\section{References}

[1] Doury A., Une méthode de calcul pratique et générale pour la prévision numérique des pollutions véhiculées par l'atmosphère, Commissariat à l'énergie atomique, Rapport CEA R 4280 (Rev 1), Saclay, France (1976), $37 \mathrm{p}$.

[2] Pasquill F., Atmospheric Diffusion, $2^{\text {nd }}$ edition, Ellis Horwood Ed., Londres (1974).

[3] Briggs G. A., Rural dispersion parameters. In: Boubel, R.W., Fox D.L., Turner, D.B., Stern, A.C., Academic Press (Eds), Fundamentals of air pollution. San Diego, California, USA (1976), pp.291-319.

[4] Maro D., Baron Y., Germain. P., Crabol B., Hebert D., Solier L., Utilisation du krypton 85 rejeté dans l'environnement par l'usine de retraitement de La Hague comme outil d'étude de la dispersion atmosphérique : Bilan de 14 campagnes de mesures, Institut de Protection et de Sûreté Nucléaire, Rapport DPRE/SERNAT/99-14, Fontenay aux roses, France (1999) $18 \mathrm{p}$.

[5] Lagoutine F., Cousol N., Legrand J., Table des radionucléides Volume 2, Commissariat à l'Energie Atomique, Bureau National de Métrologie, Laboratoire de Métrologie des Rayonnements Ionisants, Paris (1984).

[6] Pannetier R., Distribution, transfert atmosphérique et bilan de krypton 85, Commissariat à l'énergie atomique, Rapport CEA R 3591, Saclay, France (1968), 177 p. 
[7] Caire ; Système 2SNM, Guide d'exploitation, volume 1, Cherbourg, France (1996).

[8] Crabol B., Méthodes d'évaluation de la dispersion des gaz et des aérosols dans l'atmosphère, support de cours, Fontenay aux roses, France (1996) 53 p.

[9] GRNC., Modèles de transfert des radionucléides dans l'environnement, Groupe radioécologie Nord Cotentin, France (1999), volume 3, pp. 50 - 63.

[10] Hill R.A., Lowles I., Teasdale I., Chambers N., Puxley C., Parker T., Comparison between field measurements of ${ }^{85} \mathrm{Kr}$ around the BNFL Sellafield reprocessing plant and the predictions of the NRPB R91 and UK-ADMS atmospheric dispersion models. Proceeding of the sixth international conference on harmonisation within atmospheric dispersion modelling for regulatory purposes (1999). Paper number 86. [11] Maro D., Crabol B., Germain P., Hebert D., Etude de la dispersion en champ proche pour des rejets en hauteur, Institut de Protection et de Sûreté Nucléaire, Rapport DPRE/SERNAT/99-16, Fontenay aux roses, France (1999) 16 p. 